\section{BMJ Open} Ophthalmology

\title{
Postoperative endophthalmitis with and without intracameral moxifloxacin prophylaxis in a high volume surgery setting
}

\author{
Subash Bhatta (D) , ${ }^{1}$ Nayana Pant, ${ }^{2}$ Manish Poudel ${ }^{3}$
}

To cite: Bhatta S, Pant N, Poudel M. Postoperative endophthalmitis with and without intracameral moxifloxacin prophylaxis in a high volume surgery setting. BMJ Open Ophthalmology 2021;6:e000609. doi:10.1136/ bmjophth-2020-000609

Received 30 August 2020 Accepted 21 May 2021

\section{Check for updates}

(C) Author(s) (or their employer(s)) 2021. Re-use permitted under CC BY-NC. No commercial re-use. See rights and permissions. Published by BMJ.

${ }^{1}$ Retina, Geta Eye Hospital, Kailali, Nepal

${ }^{2}$ Cataract, Geta Eye Hospital, Kailali, Nepal

${ }^{3}$ Statistics, Tilganga Institute of Ophthalmology, Kathmandu, Nepal

Correspondence to Subash Bhatta; subashbhatta@ gmail.com

\section{ABSTRACT}

Objective This study was conducted to understand the effects of intracameral moxifloxacin in decreasing the incidence of postoperative endophthalmitis after cataract surgery in an eye hospital with a high volume surgical load. Methods and analysis In this single-centre, retrospective, clinical registry-based study, we compared the rates of postoperative endophthalmitis in 31340 cataract surgery patients operated during 22 months after June 2018 who received intracameral moxifloxacin to 80 643 patients operated during 41 months before June 2018 who did not receive intracameral moxifloxacin. All patients received subconjunctival gentamycin and dexamethasone. Combined surgical procedures were excluded from the study.

Results There was a significant reduction $(p<0.001)$ of postoperative endophthalmitis rates in cataract surgeries from $0.144 \%$ (116/80 643$)$ to $0.025 \%$ (8/31 340) after initiation of intracameral moxifloxacin. Endophthalmitis rates decreased from $0.120 \%$ (12/9942) to $0.009 \%$ (1/10 787 ) in phacoemulsification group and from $0.147 \%$ (104/70 701) to $0.034 \%$ (7/20 553) in manual smallincision cataract surgeries. Gram-positive organisms including Coagulase-negative staphylococci $(37.9 \%, n=11)$ and Staphylococcus aureus (S. aureus $34.5 \%, \mathrm{n}=10$ ) were the most common organisms isolated out of 29 culturepositive cases. $24.1 \%$ (28/116) endophthalmitis cases in group without moxifloxacin were culture positive compared with $14.3 \%(1 / 7)$ of cases in moxifloxacin group. $72 \%$ $(\mathrm{n}=8)$ of the Coagulase-negative staphylococci and $80 \%$ of $S$. aureus isolates $(\mathrm{n}=8)$ showed in vitro sensitivity to moxifloxacin.

Conclusion Prophylactic use of intracameral moxifloxacin injection in addition to subconjunctival gentamycin in cataract surgery is associated with a significant decrease in rates of postoperative endophthalmitis when compared with the use of subconjunctival gentamycin alone in high volume settings.

\section{INTRODUCTION}

Improvement in operating environments, surgical techniques and use of various endophthalmitis prophylaxis measures have seen the rates of postoperative endophthalmitis decline steadily since the 1970 s as compared with very high rates seen in the early

\section{Key messages}

What is already known about this subject?

- Intracameral antibiotic injection has not been incorporated as the standard of care in cataract surgeries uniformly all over the globe as there are still issues about the role and availability of different antibiotics suitable for intracameral use.

\section{What are the new findings?}

- This study shows that cost-effective commercial preparation of intracameral (IC) moxifloxacin can significantly decrease the rates of postoperative endophthalmitis even in a high-volume surgical setting where instruments are sterilised and reused for cataract surgeries. There was also a threefold reduction in rates of endophthalmitis in cases with posterior capsular rupture and vitreous loss with the use of IC moxifloxacin.

\section{How might these results change the focus of} research or clinical practice?

IC moxifloxacin injection can be incorporated as the standard of care in cataract surgeries performed in low-cost high-volume cataract surgery centres in developing countries as it may be the most costeffective way to reduce the rates of postoperative endophthalmitis in such settings.

20th century. ${ }^{1-6}$ Findings from the European Society of Cataract and Refractive Surgeons (ESCRS) group and subsequent other studies have helped to establish the benefits of intracameral antibiotics for endophthalmitis prophylaxis in recent years. ${ }^{7-13}$ Among various antibiotics used for endophthalmitis prophylaxis, cefuroxime ( $82 \%$ ) was the most commonly used intracameral antibiotic in Europe, whereas ASCRS survey 2014 showed that vancomycin $(37 \%)$ and moxifloxacin (33\%) were the preferred choices in the USA. ${ }^{1415}$ Compared with the other two drugs, moxifloxacin has broader antimicrobial coverage and the required intracameral dose has a good safety profile. ${ }^{16}$ Though there is no level I evidence for the use of intracameral 
moxifloxacin, various retrospective reports have given some assurance of its effectiveness and safety profile. ${ }^{16-21}$ In a couple of studies including more than two million consecutive cataract cases, Aravind Hospital and its allied centres in India reported a reduction of endophthalmitis rates from $0.07 \%$ to $0.02 \%$ with the use of intracameral moxifloxacin. ${ }^{20}{ }^{21}$ Using the same protocol based on their published work, Geta Eye Hospital, a tertiary level eye hospital in Nepal performing high volume cataract surgeries, instituted intracameral moxifloxacin as routine endophthalmitis prophylaxis for cataract surgeries since June 2018. This study evaluates the endophthalmitis rates in cataract surgeries done between June 2018 and March 2020 when intracameral antimicrobial (moxifloxacin) was given to all cataract surgery cases in addition to the subconjunctival injection of gentamycin and dexamethasone as compared with the infection rates between January 2015 and May 2018 when subconjunctival injection of gentamycin and dexamethasone was only used for endophthalmitis prophylaxis.

\section{MATERIALS AND METHODS}

This is a single-centre, retrospective, clinical registry-based study, done at Geta Eye Hospital, Nepal. We reviewed cataract surgery and follow-up records from January 2015 to March 2020. All cataract surgeries done in secondary level hospitals, eye care centres and outreach camps were excluded from the study to ensure the uniformity of all other parameters related to surgery. Combined glaucoma surgeries and any cataract surgeries combined with corneal and vitreoretinal procedures were excluded as they carry a different level of risk for endophthalmitis compared with routine cataract surgeries. A total of 113 044 patients were operated on for cataracts in the base hospital during this period. After applying the exclusion criteria, 111983 cases were included in the study. No intracameral antibiotics were used before June 2018, whereas all cataract cases received intracameral moxifloxacin after that period. A total of 31340 patients were included in the intracameral antibiotics group and 80 643 in the other group, who did not receive intracameral moxifloxacin after surgery. Both the groups received a subconjunctival injection of gentamycin $(20 \mathrm{mg} / 0.5 \mathrm{~mL})$ and dexamethasone $(2 \mathrm{mg} / 0.5 \mathrm{~mL})$ at the end of cataract surgery. As there were no other major changes in operating theatre protocols and surgical technique from the earlier study years, we assumed that the change in rates of endophthalmitis over these 22 months window was reflective of the effects of intracameral moxifloxacin.

Operating rooms were completely mopped and cleaned with disinfectant every day. Any waste or soiled surfaces were cleaned between surgeries and disinfected as necessary. Operation theatre (OT) disinfection was done with novacide (3\% w/v Poly (hexamethylene biguanide) hydrochloride $+10 \% \mathrm{w} / \mathrm{v}$ Didecyl dimethyl ammonium chloride) at least once or more in a week if required. The instruments, eye towels and table cloths used for surgery were sterilised using an autoclave.
All cataracts were operated by ophthalmologists or ophthalmology residents under the observation of a senior surgeon. Senior surgeons generally used two operating tables at a time to minimise the turnover time between surgeries to manage the high cataract volumes. Junior doctors used single operating tables. The surgeon and assistant changed their gloves after each case. The cataract set containing the instruments for the surgery was also changed after each case. Simcoe irrigation aspiration cannula was changed after each case but the irrigating fluid remained the same. Phaco tips were also reused in case of phacoemulsification surgeries. Manual small-incision cataract surgery (MSICS) incisions were sutured only if wound integrity was deemed inadequate at the end of the surgery due to any cause including complications in sclerocorneal tunnel construction.

Surgeries were done under peribulbar block or topical anaesthesia. No topical antibiotics were used before surgery. The periocular area was cleaned with $5 \%$ povidone-iodine solution in the block room before patients were taken to the operating table. The periocular area and conjunctival sac were again cleaned with povidone-iodine solution and the eye was draped with sterile eye towels. All aseptic precautions were taken during surgery. Most of the MSICS cases were done using superior incision and all phacoemulsification cases were performed via a temporal clear corneal incision. After the end of the surgery, $0.1 \mathrm{~mL}(0.5 \mathrm{mg})$ of moxifloxacin was injected into the intracameral space. It was followed by subconjunctival injection $(0.5 \mathrm{~mL})$ of gentamycin and dexamethasone combination. The eyes were padded and evaluated the next day. Ofloxacin $(0.3 \%)$ eye drops were prescribed for the postoperative period of 1 month at a dosage of 4-6 times per day. Steroid eye drops (prednisolone acetate $1 \%$ or dexamethasone $0.1 \%$ ) were prescribed four times a day to hourly dosage according to the level of postoperative inflammation and were tapered after a week or two.

Commercially available intracameral moxifloxacin formulation (Auromox), manufactured by Aurolab, India was used in all intracameral antibiotic group cases. Auromox is available in sterile glass vials containing 5 $\mathrm{mg}$ of preservative-free moxifloxacin hydrochloride in $1 \mathrm{~mL}$ of solution. The sterile vials were opened and the required dose $(0.1 \mathrm{~mL})$ of moxifloxacin (concentration of $0.5 \mathrm{mg}$ ) were drawn in sterile syringes by the surgery assistant for use by the operating surgeon.

Patients who presented with pain and/or sudden decrease in visual acuity after cataract surgery with signs of diffuse intraocular inflammation (red-eye, hypopyon, vitreous inflammation) were evaluated in the vitreoretinal department. A presumptive diagnosis of postoperative endophthalmitis was made after ruling out other causes like toxic anterior segment syndrome (TASS) and postoperative non-infectious uveitis on the basis of relevant history and clinical examination. Multiple clinical findings were considered in differentiating postoperative endophthalmitis from these entities as they often have 
overlapping patterns. History suggestive of uveitis, presence of complicated cataracts, lens-induced glaucoma or uncontrolled intraocular pressure (IOP) before surgery, and evidence of retained lens material in anterior chamber or vitreous cavity after surgery were considered supporting evidences in making a diagnosis of non-infectious postoperative uveitis. Early presentation (usually 12-48 hours after surgery) with relatively less or no pain, limbus-to-limbus corneal oedema and absence of vitritis were used as supporting evidences while making a diagnosis of TASS. Delayed presentation (generally after 2-3 days) often with significant pain, presence of more severe anterior chamber reaction with development of posterior synechiae and presence of vitritis were considered as important diagnostic criteria for postoperative endophthalmitis. B-scan ultrasonography was used to confirm vitreous involvement in cases with opaque media.

All endophthalmitis cases with onset of symptoms within 6 weeks of surgery were included in the study. Each case underwent vitreous tap and injection of intravitreal antibiotics at the first presentation and the sample was sent to the laboratory for staining and culture sensitivity. Disc diffusion by the Kirby-Bauer method was commonly used to evaluate the antibiotic sensitivity of the isolated pathogens. After a successful isolation and growth of a bacterial strain in a Muller-Hinton agar with an incubation period of about 15 hours, the microbe was reported to be susceptible to an antibiotic if the test showed an area of bacterial inhibition of at least one standard zone diameter based on the recommendation of the Clinical Laboratory Standards Institute (CLSI document M100S25; 2015).

The necessary data were retrieved from hospital electronic records and verified with clinical registers maintained in the operating theatre and clinical laboratory. Data were imported to MS excel and cleaned. Data analysis was done with SPSS V.20 and p values for differences in the two comparative group's categorical variables were calculated using the $\chi^{2}$ test or Fischer exact test wherever applicable. P-value less than 0.05 was considered to be statistically significant.

Patients or the public were not involved in the design, conduct, reporting or dissemination plans of our study.

\section{RESULTS}

A total of 80643 cataract surgery patients operated on from January 2015 to May 2018 did not receive intracameral moxifloxacin injection. Out of these cases, 70 701 cases underwent MSICS, and 9942 cases underwent phacoemulsification. Subsequent 31340 cases, including 20553 MSICS and 10787 phacoemulsification surgeries operated from June 2018 to March 2020, received intracameral moxifloxacin. There was significant reduction $(\mathrm{p}<0.001)$ of postoperative endophthalmitis rates from $0.144 \%(116 / 80643)$ to $0.025 \%(8 / 31340)$ after initiation of intracameral moxifloxacin. Both the MSICS and phacoemulsification groups showed a significant decrease in the number of postoperative endophthalmitis cases after the intervention as shown in table 1.

The yearly trend of postoperative endophthalmitis rates in cases with and without posterior capsular rupture (PCR) is shown in table 2. The rates of endophthalmitis ranged from $0.111 \%$ to $0.188 \%$ in the group without moxifloxacin and from $0.020 \%$ to $0.036 \%$ in the moxifloxacin group. Both the groups were given s/c gentamycin and dexamethasone injection after surgery. There were no endophthalmitis cases reported in the first 3 months of 2020 in the moxifloxacin group. Postoperative endophthalmitis rates increased from $0.103 \%(114 / 110131)$ in cases without PCR to $0.539 \%(10 / 1852)$ in cases with PCR and vitreous loss $(\mathrm{p}<0.001)$. Rates of endophthalmitis in cases with PCR and vitreous loss decreased from $0.663 \%$ $(9 / 1357)$ to $0.202 \%(1 / 495)$ after the use of intracameral moxifloxacin.

The majority of surgeries (92 519/106 869, 86.6\%) done over the study years were done by surgeons greater than 2 years of surgical experience. The year-wise trend of endophthalmitis rates for surgeons of less than and more than 2 years of surgical experience, over the five completed calendar years in the study, are shown in table 3. PCR rates were higher $(2.84 \%, \mathrm{n}=408 / 14350)$ for junior surgeons(less than 2 years of surgical experience) as compared with more experienced surgeons $(1.47 \%$, $\mathrm{n}=1366 / 92519)$. The overall rate of endophthalmitis was $0.279(40 / 14350)$ for surgeons less than 2 years of surgical experience which was significantly higher than

Table 1 Endophthalmitis rates in MSICS and phacoemulsification with and without intracameral moxifloxacin

\begin{tabular}{|c|c|c|c|c|c|c|}
\hline \multirow[b]{2}{*}{ Surgery type } & \multicolumn{2}{|c|}{ Without IC moxifloxacin* } & \multicolumn{2}{|c|}{ With IC moxifloxacin* } & \multirow[b]{2}{*}{$P$ valuet } & \multirow[b]{2}{*}{ OR $(95 \% \mathrm{Cl})$} \\
\hline & $\begin{array}{l}\text { Total } \\
\text { surgery }\end{array}$ & $\begin{array}{l}\text { No. of } \\
\text { endophthalmitis, } \\
\text { N (\%) }\end{array}$ & $\begin{array}{l}\text { Total } \\
\text { surgery }\end{array}$ & $\begin{array}{l}\text { No. of } \\
\text { endophthalmitis, } \\
\text { N (\%) }\end{array}$ & & \\
\hline MSICS & 70701 & $104(0.147)$ & 20553 & $7(0.034)$ & $<0.001$ & 1.21 (1.52 to 1.27$)$ \\
\hline Phaco-emulsification & 9942 & $12(0.12)$ & 10787 & $1(0.009)$ & 0.002 & $1.93(1.65$ to 2.25$)$ \\
\hline Total & 80643 & $116(0.144)$ & 31340 & $8(0.025)$ & $<0.001$ & $1.3(1.24$ to 1.36$)$ \\
\hline
\end{tabular}

*Both groups received s/c gentamycin and dexamethasone injection after surgery.

†P values were adjusted using Bonferroni corrections.

IC, intracameral; MSICS, manual small-incision cataract surgery. 
Table 2 Postoperative endophthalmitis rates over the study period with subanalysis of endophthalmitis rates in cases with and without PCR and vitreous loss

\begin{tabular}{|c|c|c|c|c|c|c|c|c|c|c|}
\hline \multirow[b]{2}{*}{ Year } & \multicolumn{5}{|c|}{ Without intracameral moxifloxacin* } & \multicolumn{4}{|c|}{ With intracameral moxifloxacin* } & \multirow[b]{2}{*}{ P value } \\
\hline & 2015 & 2016 & 2017 & $\begin{array}{l}2018 \\
\text { January-May }\end{array}$ & Total & $\begin{array}{l}2018 \\
\text { June- } \\
\text { December }\end{array}$ & 2019 & $\begin{array}{l}2020 \\
\text { January- } \\
\text { March }\end{array}$ & Total & \\
\hline Study cases & 24320 & 23591 & 22623 & 10109 & 80643 & 9702 & 16524 & 5114 & 31340 & \\
\hline POE (\%) & $27(0.111)$ & $31(0.131)$ & $39(0.172)$ & 19 (0.188) & $116(0.144)$ & $2(0.020)$ & $6(0.036)$ & $0(0.0)$ & $8(0.025)$ & $<0.001$ \\
\hline $\begin{array}{l}\text { PCR with } \\
\text { V-loss (\%) }\end{array}$ & $382(1.57)$ & 385 (1.63) & $416(1.84)$ & $174(1.72)$ & 1357 (1.68) & 153 (1.58) & $264(1.60)$ & 78 (1.52) & 495 (1.58) & \\
\hline POE (\%) & $2(0.523)$ & $2(0.519)$ & $3(0.721)$ & $2(1.149)$ & $9(0.663)$ & $0(0.0)$ & $1(0.379)$ & $0(0.0)$ & $1(0.202)$ & 0.61 \\
\hline PCR w/o V-loss & 23938 & 23206 & 22207 & 9935 & 79286 & 9549 & 16260 & 5036 & 30485 & \\
\hline POE (\%) & $25(0.104)$ & $29(0.125)$ & $36(0.162)$ & $17(0.171)$ & $107(0.135)$ & $2(0.020)$ & $5(0.030)$ & $0(0.0)$ & $7(0.023)$ & $<0.001$ \\
\hline
\end{tabular}

*Both groups received s/c gentamycin and dexamethasone injection after surgery.

†P values for difference in postoperative endophthalmitis rates between total cases in the two groups (with and without intracameral moxifloxacin). $P$ values were adjusted using Bonferroni corrections

PCR, posterior capsular rupture; POE, postoperative endophthalmitis; V-loss, vitreous loss.

the endophthalmitis rate of $0.091 \%$ (84/92 519) seen for surgeons with greater than 2 years of surgical experience.

As shown in table 4, Gram-positive organisms especially Coagulase-negative staphylococci (CONS, $n=11,37.9 \%$ ) and Staphylococcus aureus (S. aureus, $34.5 \%, \mathrm{n}=10$ ) were the most common organisms isolated out of 29 culturepositive cases. $24.1 \%(28 / 116)$ of endophthalmitis cases in the group without moxifloxacin were culture positive as compared with $14.3 \%(1 / 7)$ of cases in the moxifloxacin group. Out of 21 cases of Staphylococci species, $72 \%$ $(n=8)$ of the CONS and $80 \%$ of $S$. aureus isolates $(n=8)$ showed in vitro sensitivity to moxifloxacin when zone of inhibition of $28 \mathrm{~mm}$ and $24 \mathrm{~mm}$, respectively, were considered as susceptible breakpoints for moxifloxacin disc content of $5 \mathrm{ug}$. Gentamycin was effective against $27 \%(\mathrm{n}=3)$ of CONS and $50 \%(\mathrm{n}=5)$ of $S$. aureus when the susceptible break point of $15 \mathrm{~mm}$ for $10 \mathrm{ug}$ disc content was used as reference.

Rates of postoperative endophthalmitis with and without intracameral antibiotics as reported by comparative studies from different countries are shown in table 5. All these studies show a trend of decreasing endophthalmitis rates with the use of intracameral antibiotic prophylaxis.

\section{DISCUSSION}

Commercially available intracameral antibiotic formulations have a distinctive advantage in alleviating surgeons' concern about inappropriate dilution causing TASS or other adverse reactions in the eye. Moxifloxacin (Auromax, Aurolab, India) is the only commercial intracameral formulation available in the country. There are no randomised clinical trials to prove the efficacy and safety of intracameral moxifloxacin but some retrospective studies have indicated that this intervention is effective in decreasing the rates of endophthalmitis in cataract surgery patients. ${ }^{17-21}$ As per best of our knowledge, baring the reports from Aravind Hospitals India, whose sister organisation produces commercial intracameral moxifloxacin; this is perhaps the largest single-centre study reporting on the results of intracameral moxifloxacin as endophthalmitis prophylaxis.

In this study, a total of 31340 patients who were operated on after June 2018 and received intracameral

Table 3 Year-wise trends of PCR and endophthalmitis rates in cataract surgeries done by surgeons of less than and more than 2 years of surgical experience

\begin{tabular}{|c|c|c|c|c|c|c|c|c|}
\hline \multirow[b]{2}{*}{ Year } & \multirow[b]{2}{*}{ Total surgery } & \multicolumn{3}{|c|}{ Surgical experience $>2$ years } & \multicolumn{3}{|c|}{ Surgical experience $<2$ years } & \multirow[b]{2}{*}{ P value } \\
\hline & & $\begin{array}{l}\text { No. of } \\
\text { surgery }\end{array}$ & $\begin{array}{l}\text { PCR rates } \\
\text { N (\%) }\end{array}$ & $\begin{array}{l}\text { No. of POE, } \\
(\%)\end{array}$ & No. of surgery & $\begin{array}{l}\text { PCR rates } \\
\text { N (\%) }\end{array}$ & $\begin{array}{l}\text { No. of POE } \\
(\%)\end{array}$ & \\
\hline 2015 & 24320 & 23464 & $351(1.49)$ & $22(0.094)$ & 856 & 31 (3.62) & $5(0.584)$ & 0.01 \\
\hline 2016 & 23591 & 22231 & 347 (1.56) & $26(0.117)$ & 1360 & 38 (2.79) & $5(0.368)$ & 0.155 \\
\hline 2017 & 22623 & 16421 & $252(1.53)$ & $15(0.091)$ & 6202 & $164(2.64)$ & $24(0.387)$ & $<0.001$ \\
\hline 2018 & 19811 & 16453 & 235 (1.43) & $17(0.103)$ & 3358 & $92(2.74)$ & $4(0.119)$ & 3.855 \\
\hline 2019 & 16524 & 13950 & $181(1.30)$ & $4(0.029)$ & 2574 & $83(3.22)$ & $2(0.078)$ & 1.185 \\
\hline Total & 106869 & 92519 & $1366(1.47)$ & $84(0.091)$ & 14350 & $408(2.84)$ & $40(0.279)$ & $<0.001$ \\
\hline
\end{tabular}

$P$ values were adjusted using Bonferroni corrections.

${ }^{*} P$ value: $p$ values for $P O E$ rates between surgeons with less than and more than 2 years of surgical experience.

PCR, posterior capsular rupture; POE, postoperative endophthalmitis. 
Table 4 Culture and antibiotic sensitivity results of vitreous samples of endophthalmitis cases

\begin{tabular}{|c|c|c|c|c|c|c|}
\hline \multirow[b]{2}{*}{ Culture results } & \multicolumn{2}{|c|}{ Intracameral moxifloxacin } & \multicolumn{4}{|c|}{ Susceptibility to antibiotics } \\
\hline & No & Yes & $\begin{array}{l}\text { Moxifloxacin } \\
\text { N (\%) }\end{array}$ & $\begin{array}{l}\text { Ceftazidime } \\
\text { N (\%) }\end{array}$ & $\begin{array}{l}\text { Vancomycin } \\
\mathrm{N}(\%)\end{array}$ & $\begin{array}{l}\text { Gentamycin } \\
\mathrm{N}(\%)\end{array}$ \\
\hline CONS & 11 & 0 & $8(72.8)$ & $6(54.5)$ & $10(90.1)$ & $3(27)$ \\
\hline Staphylococcus aureus & 9 & 1 & $8(80)$ & $5(50)$ & $9(90)$ & $5(50)$ \\
\hline Streptococcus species & 3 & 0 & $3(100)$ & $2(66.6)$ & $3(100)$ & $2(66.6)$ \\
\hline Haemophilus species & 2 & 0 & $2(100)$ & $2(100)$ & *- & $1(50)$ \\
\hline Klebsiella species & 1 & 0 & $1(100)$ & $1(100)$ & *- & $1(100)$ \\
\hline Proteus species & 1 & 0 & $1(100)$ & $1(100)$ & *- & $1(100)$ \\
\hline Clostridium species & 1 & 0 & $1(100)$ & 0 & $1(100)$ & *- \\
\hline No growth & 88 & 7 & - & - & - & - \\
\hline Total & 116 & 8 & - & - & - & - \\
\hline
\end{tabular}

Susceptible break points for major isolated organisms (Clinical Laboratory Standards Institute guidelines): Moxifloxacin (5 ug): CONS 28 mm, Staphylococcus aureus 24 mm, Streptococcus sp. 18 mm; Ceftazidime (30 ug): CONS 25 mm, S. aureus 22 mm; Streptococcus sp. 24 mm; Vancomycin: CONS 4 ug/mL (MIC), S. aureus 2 ug/mL (MIC); Streptococcus sp. 17 mm (30 ug); Gentamycin (10 ug): CONS 15 mm, S. aureus: $15 \mathrm{~mm}$, Streptococcus sp. $15 \mathrm{~mm}$.

*In-vitro sensitivity tests for these antibiotics were not performed.

CONS, coagulase-negative staphylococci; MIC, minimum inhibitory concentration.

moxifloxacin could be compared with a pool of 80643 patients who were operated on before that time frame and received no intracameral antibiotics. Both the groups received a subconjunctival injection of gentamycin and dexamethasone at the end of cataract surgery. Subconjunctival gentamycin injection was considered for endophthalmitis prophylaxis since early days in the hospital as it was the most economical and readily available antimicrobial agent known to have bactericidal action against some common pathogens causing postoperative endophthalmitis. We continued using subconjunctival gentamycin along with intracameral moxifloxacin as we could not risk removing a long-used endophthalmitis prophylaxis method without confirming the additional benefits of intracameral moxifloxacin in reducing the rates of endophthalmitis, especially when dealing with a high volume of surgical cases. We used the same postoperative antibiotic regimen of Ofloxacin $0.3 \%, 4-6$

Table 5 Comparative studies showing endophthalmitis rates with and without intracameral antibiotics

\begin{tabular}{|c|c|c|c|c|c|c|}
\hline \multirow[b]{2}{*}{ Country } & \multirow[b]{2}{*}{ IC antibiotic used } & \multicolumn{2}{|c|}{ With IC antibiotic } & \multicolumn{2}{|c|}{ Without IC antibiotic } & \multirow[b]{2}{*}{ Reference } \\
\hline & & $\begin{array}{l}\text { No. of } \\
\text { surgeries }\end{array}$ & POE \% (N) & $\begin{array}{l}\text { No. of } \\
\text { surgeries }\end{array}$ & POE \% (N) & \\
\hline Sweden & Cefuroxime & 223156 & $0.045(100)$ & 2315 & $0.350(8)$ & Lundstorm et $a^{24}$ \\
\hline 9 countries* & Cefuroxime & 6836 & $0.073(5)$ & 6862 & $0.335(23)$ & ESCRS study $^{7}$ \\
\hline France & Cefuroxime & 2289 & $0.044(1)$ & 2826 & $1.238(35)$ & Barreau et $a l^{8}$ \\
\hline USA & $\begin{array}{l}\text { Cefuroxime†/ } \\
\text { moxifloxacin/ } \\
\text { vancomycin }\end{array}$ & 7108 & $0.014(1)$ & 2878 & $0.313(9)$ & Shorstein et $a l^{11}$ \\
\hline Sweden & $\begin{array}{l}\text { Cefuroxime/ } \\
\text { moxifloxacin }\end{array}$ & 461951 & $0.027(123)$ & 2804 & $0.39(11)$ & Friling et $a l^{9}$ \\
\hline Singapore & Cefazolin & 20638 & $0.01(2)$ & 29539 & $0.064(19)$ & Tan et $a l^{25}$ \\
\hline Japan & Moxifloxacin & 18794 & $0.01(3)$ & 15958 & $0.05(8)$ & Matsuura et al ${ }^{17}$ \\
\hline Spain & Cefuroxime & 12868 & $0.039(5)$ & 6595 & $0.59(35)$ & $\begin{array}{l}\text { Rodriguez-Carvacea } \\
\text { et } a^{10}\end{array}$ \\
\hline Iran & Cefuroxime & 25920 & $0(0)$ & 193440 & $0.014(28)$ & Jabbarvand et al ${ }^{12}$ \\
\hline India & Moxifloxacin & 315383 & $0.02(68)$ & 303244 & $0.07(218)$ & Haripriya et $a l^{20}$ \\
\hline Nepal & Moxifloxacinł & 31340 & $0.025(8)$ & 80643 & $0.144(116)$ & Current study \\
\hline
\end{tabular}

${ }^{*}$ Countries include Austria, Belgium, Germany, Italy, Poland, Portugal, Spain, Turkey and UK.

†IC moxifloxacin or IC vancomycin was substituted for cefuroxime in case of suspected allergy to cefuroxime.

¥IC moxifloxacin was used along with subconjunctival gentamycin injection.

ESCRS, European Society of Cataract and Refractive Surgeons; IC, intracameral; POE, postoperative endophthalmitis. 
times per day in both the groups as we were interested in studying the effects of intracameral moxifloxacin in reducing postoperative endophthalmitis when other variables were similar. Moxifloxacin eye drops were not previously available in Nepal and are still significantly more expensive than Ofloxacin eye drops.

Our postoperative endophthalmitis rates were $0.144 \%$ $(0.111 \%-0.188 \%)$ over the 41 months before the use of intracameral moxifloxacin prophylaxis when subconjunctival gentamycin alone was used. The incidence rate of endophthalmitis without intracameral antibiotics in our study is comparable to the reports from Nepal, India and France, ${ }^{1922}{ }_{23}$ lower than endophthalmitis rates reported from some European countries and the USA, ${ }^{7-1124}$ but higher than that reported from studies coming from Singapore and pooled endophthalmitis rates from the USA. ${ }^{25} 26$ This suggests that the reported rates of endophthalmitis vary widely among different surgical centres, geographical areas and populations. The rates of endophthalmitis decreased significantly from $0.144 \%$ to $0.025 \%$ ( $p$ value $<0.001$ ) after the use of intracameral moxifloxacin along with subconjunctival gentamycin. There was a 5.6-fold reduction in endophthalmitis rate for all surgeries with addition of intracameral moxifloxacin prophylaxis. The reduction in endophthalmitis was 13-fold for phacoemulsification cases and 4.3-fold for MSICS cases. The rate of postoperative endophthalmitis in this group is comparable to the findings of ESCRS in a similar group with intracameral cefuroxime and no preoperative antibiotics. Incidence rates of endophthalmitis after the use of intracameral moxifloxacin and subconjunctival gentamycin are also comparable to few other retrospective studies reporting endophthalmitis rates of $0.01 \%-0.06 \%$ after use of intracameral antibiotics. $^{7-13} 17-2127$

The rates of endophthalmitis were lower for phacoemulsification cases compared with MSICS in both the groups (with and without intracameral moxifloxacin), but the difference was not clinically significant. A study from India showed a comparable rate of postoperative endophthalmitis in MSICS and phacoemulsification cases. ${ }^{20}$ Complicated cases are preferably operated using the MSICS technique in our practice. Greater chances of having complications and longer operating times in these complicated cases might have contributed to higher endophthalmitis rates in MSICS compared with phacoemulsification. One of the risk factors of endophthalmitis in MSICS might be the larger wound size (around $6 \mathrm{~mm}$ ) required in this technique.

When surgical data were analysed for five completed calendar years in the study, we found a significant increase in endophthalmitis rates for surgeons having less than 2 years of surgical experience $(0.279 \%, n=40 / 14350)$ as compared with those having greater than 2 years of experience $(0.091 \%, \mathrm{n}=84 / 92519)$. A study from the USA analysing the national database from the years 2003 and 2004 also showed that the risks of postoperative endophthalmitis were higher for low-volume and inexperienced surgeons. ${ }^{28}$ We presume that surgeons with lesser experience have greater complication rates and need more surgical time for each case, which may lead to higher endophthalmitis rates. In our study, PCR (with vitreous loss) rate was $1.65 \%$ ( $\mathrm{n}=1852 / 111$ 983) for overall patients and it was higher $(2.84 \%)$ for less experienced surgeons compared with that of more experienced surgeons $(1.47 \%)$. There was a nearly fivefolds increase in rates of endophthalmitis in cases with PCR. A study from Aravind Eye Hospital and its allied eye centres in India showed that PCR increased the risk of postoperative endophthalmitis by seven-folds. ${ }^{21}$ Other studies have shown that surgical complications increase the risks of postoperative endophthalmitis, but there is a paucity of literature on the relation of surgical time and postoperative endophthalmitis. ${ }^{79} 121320$ Although endophthalmitis rates for junior surgeons were higher in each of the study years, the difference was not significant in last 2 years when intracameral moxifloxacin was in use. The use of intracameral moxifloxacin decreased the rates of postoperative endophthalmitis by 3.3-folds (from $0.663 \%$ to $0.202 \%$ ) in cases with PCR and vitreous loss. These findings suggest that intracameral moxifloxacin may also be effective in decreasing the risks of endophthalmitis in cataract surgeries done by novice surgeons who are expected to have a higher number of complications and longer surgical time, increasing the risk of endophthalmitis. As high-volume centres in developing countries have a constant influx of new surgeons, joining these centres for short periods to improve their surgical skills, the use of intracameral moxifloxacin can act as an important safety cover for possible higher endophthalmitis rates associated with less experienced surgeons.

With the use of Blood Agar, MacConkey Agar, Brain Heart Infusion Broth and Saboraud dextrose Agar as culture mediums, causative organisms were isolated in $24 \%$ of cases as compared with $69 \%$ in Endophthalmitis Vitrectomy Study (EVS) and 48\% in ESCRS study. As the vitreous sample was collected with needle tap in all cases, an insufficient vitreous sample might have contributed to decreased culture-positive rates in our study. Grampositive organisms constituted $86 \%$ of culture-positive cases among which Coagulase-negative staphylococci $(\mathrm{n}=11)$ and $S$. aureus $(\mathrm{n}=10)$ were the most common isolates. Only one $(12.5 \%)$ out of eight endophthalmitis cases seen in the intracameral moxifloxacin group was culture-positive and $S$. aureus was isolated in culture. Overall, $30 \%$ of culture-proven endophthalmitis cases were gram-negative isolates, no gram-negative growth was seen in the moxifloxacin group. Antibiotic sensitivity results in our study showed that all gram negative isolates and majority of gram positive bacteria including CONS $(72.8 \%)$, and $S$. aureus $(80 \%)$ isolates were susceptible to moxifloxacin. More microbial isolates were susceptible to moxifloxacin compared with gentamycin or other drugs used in antimicrobial sensitivity tests. Thus, moxifloxacin having a broad spectrum of activity and a concentrationdependent action mechanism; intracameral use of 
this drug can help in reducing the risks of postoperative endophthalmitis in cataract surgery cases. ${ }^{29}$ There were no adverse reactions, notably no cases of TASS or endothelial decompensation, attributed to intracameral moxifloxacin in our study.

Our study has its limitations in comparing two data sets with marked differences in sample sizes. Being a retrospective study comparing data in two different time periods, we also cannot completely rule out the effects of other variables in the changes in rates of endophthalmitis noted in our study. However, we expect these effects to be minimal as there was no major change in surgical sterilisation and operative protocols over these years. The introduction of new surgeons during the study period might also have had some effect on endophthalmitis rates but their effect was almost equally distributed between the two groups as a similar percentage of cases were operated by junior surgeons in each group (with and without moxifloxacin). Some secondary data like PCR rates and surgeons' experience collected from different sources (Clinical registers in OT and hospital laboratory) were in tabular form and not in individual information. This limitation in our data set prevented us to perform interaction analysis between different parameters in the development of postoperative endophthalmitis.

As a high-volume centre in a lower-middle-income country performing cataract surgeries with a majority of reusable sterilised instruments, our endophthalmitis rates with intracameral moxifloxacin prophylaxis, being comparable to international standards, is a remarkable finding. Though we provide cataract surgery at a cost as low as 27 US\$, we have been able to incorporate intracameral moxifloxacin in all of our cataract surgeries without additional surgical charge, achieving significant safety for our patients. These findings can be very encouraging for many hospitals around the world where they still have to find the right balance between the cost of surgery and the safety of patients while operating on a large volume of cataract cases. As level I evidence for endophthalmitis prophylaxis is rare, operational results with intracameral moxifloxacin will help to build evidence about the benefits or any risks relating to this intervention.

On the basis of our study findings, we recommend the use of intracameral moxifloxacin or equivalent antimicrobial injection in addition to subconjunctival gentamicin as endophthalmitis prophylaxis in cataract surgeries, especially in high volume cataract surgery settings.

Acknowledgements We would like to acknowledge Dr Suresh Raj Pant, Chief medical director of Geta Eye Hospital for facilitating the study and Mr Bhagiram Chaudhary and Mrs Shanti Baduwal for their support in data collection.

Contributors SB and NP contributed to the design and implementation of the research, to the analysis of the results and to the writing of the manuscript. MP contributed to the design of the research, analysis of the results and the writing of the manuscript.

Funding The authors have not declared a specific grant for this research from any funding agency in the public, commercial or not-for-profit sectors.

Competing interests None declared.
Patient and public involvement Patients and/or the public were not involved in the design, or conduct, or reporting or dissemination plans of this research.

Patient consent for publication Not required.

Ethics approval Ethical approval was taken from the institutional review committee of Nepal Netra Jyoti Sangh (NNJS) and the study was conducted as per the principles outlined in the Declaration of Helsinki.

Provenance and peer review Not commissioned; externally peer reviewed.

Data availability statement All data are available upon request.

Open access This is an open access article distributed in accordance with the Creative Commons Attribution Non Commercial (CC BY-NC 4.0) license, which permits others to distribute, remix, adapt, build upon this work non-commercially, and license their derivative works on different terms, provided the original work is properly cited, appropriate credit is given, any changes made indicated, and the use is non-commercial. See: http://creativecommons.org/licenses/by-nc/4.0/.

ORCID iD

Subash Bhatta http://orcid.org/0000-0001-9155-0195

\section{REFERENCES}

1 West ES, Behrens A, McDonnell PJ, et al. The incidence of endophthalmitis after cataract surgery among the U.S. Medicare population increased between 1994 and 2001. Ophthalmology 2005;112:1388-94.

2 Taban M, Behrens A, Newcomb RL, et al. Acute endophthalmitis following cataract surgery: a systematic review of the literature. Arch Ophthalmol 2005;123:613-20.

3 Ciulla TA, Starr MB, Masket S. Bacterial endophthalmitis prophylaxis for cataract surgery: an evidence-based update. Ophthalmology 2002;109:13-24.

4 Ferguson AW, Scott JA, McGavigan J, et al. Comparison of $5 \%$ povidone-iodine solution against $1 \%$ povidone-iodine solution in preoperative cataract surgery antisepsis: a prospective randomised double blind study. Br J Ophthalmol 2003;87:163-7.

5 Carrim ZI, Mackie G, Gallacher G, et al. The efficacy of 5\% povidone-iodine for 3 minutes prior to cataract surgery. Eur $J$ Ophthalmol 2009;19:560-4.

6 Quiroga LP, Lansingh V, Laspina F, et al. A prospective study demonstrating the effect of $5 \%$ povidone-iodine application for anterior segment intraocular surgery in Paraguay. Arq Bras Oftalmol 2010;73:125-8.

7 Endophthalmitis Study Group, European Society of Cataract \& Refractive Surgeons. Prophylaxis of postoperative endophthalmitis following cataract surgery: results of the ESCRS multicenter study and identification of risk factors. J Cataract Refract Surg 2007;33:978-88.

8 Barreau G, Mounier M, Marin B, et al. Intracameral cefuroxime injection at the end of cataract surgery to reduce the incidence of endophthalmitis: French study. J Cataract Refract Surg 2012;38:1370-5.

9 Friling E, Lundström M, Stenevi U, et al. Six-Year incidence of endophthalmitis after cataract surgery: Swedish national study. $J$ Cataract Refract Surg 2013;39:15-21.

10 Rodríguez-Caravaca G, García-Sáenz MC, Villar-Del-Campo MC, et al. Incidence of endophthalmitis and impact of prophylaxis with cefuroxime on cataract surgery. J Cataract Refract Surg 2013;39:1399-403.

11 Shorstein NH, Winthrop KL, Herrinton LJ. Decreased postoperative endophthalmitis rate after institution of intracameral antibiotics in a northern California eye department. J Cataract Refract Surg 2013;39:8-14.

12 Jabbarvand M, Hashemian $\mathrm{H}$, Khodaparast M, et al. Endophthalmitis occurring after cataract surgery: outcomes of more than 480 000 cataract surgeries, epidemiologic features, and risk factors. Ophthalmology 2016;123:295-301.

13 Daien V, Papinaud L, Gillies MC, et al. Effectiveness and safety of an intracameral injection of cefuroxime for the prevention of endophthalmitis after cataract surgery with or without perioperative capsular rupture. JAMA Ophthalmol 2016;134:810-6.

14 Barry P. Adoption of intracameral antibiotic prophylaxis of endophthalmitis following cataract surgery: update on the ESCRS endophthalmitis study. J Cataract Refract Surg 2014;40:138-42.

15 Chang DF, Braga-Mele R, Henderson BA, et al. Antibiotic prophylaxis of postoperative endophthalmitis after cataract surgery: results of the 2014 ASCRS member survey. J Cataract Refract Surg 2015;41:1300-5. 
16 Libre PE, Mathews S. Endophthalmitis prophylaxis by intracameral antibiotics: In vitro model comparing vancomycin, cefuroxime, and moxifloxacin. J Cataract Refract Surg 2017;43:833-8.

17 Matsuura K, Miyoshi T, Suto C, et al. Efficacy and safety of prophylactic intracameral moxifloxacin injection in Japan. J Cataract Refract Surg 2013;39:1702-6.

18 Arshinoff SA, Modabber M. Dose and administration of intracameral moxifloxacin for prophylaxis of postoperative endophthalmitis. $J$ Cataract Refract Surg 2016;42:1730-41.

19 Haripriya A, Chang DF, Namburar S, et al. Efficacy of intracameral moxifloxacin endophthalmitis prophylaxis at Aravind eye hospital. Ophthalmology 2016;123:302-8.

20 Haripriya A, Chang DF, Ravindran RD. Endophthalmitis reduction with intracameral moxifloxacin prophylaxis: analysis of 600000 surgeries. Ophthalmology 2017;124:768-75.

21 Haripriya A, Chang DF, Ravindran RD. Endophthalmitis reduction with intracameral moxifloxacin in eyes with and without surgical complications: results from 2 million consecutive cataract surgeries. $J$ Cataract Refract Surg 2019;45:1226-33.

22 Bhatta S, Patel PJ, Awasthi S, et al. Visual outcomes of high-volume compared with low-volume manual small-incision cataract surgery in Nepal. J Cataract Refract Surg 2020;46:1119-25.

23 Creuzot-Garcher C, Benzenine E, Mariet A-S, et al. Incidence of acute postoperative endophthalmitis after cataract surgery: a nationwide study in France from 2005 to 2014. Ophthalmology 2016;123:1414-20.

24 Lundström M, Wejde G, Stenevi U, et al. Endophthalmitis after cataract surgery: a nationwide prospective study evaluating incidence in relation to incision type and location. Ophthalmology 2007;114:866-70.

25 Tan CSH, Wong HK, Yang FP. Epidemiology of postoperative endophthalmitis in an Asian population: 11-year incidence and effect of intracameral antibiotic agents. J Cataract Refract Surg 2012;38:425-30.

26 Pershing S, Lum F, Hsu S, et al. Endophthalmitis after cataract surgery in the United States: a report from the intelligent research in sight registry, 2013-2017. Ophthalmology 2020;127:151-8.

27 Vieira IV, Boianovsky C, Saraiva TJ, et al. Safety and efficacy of intracameral moxifloxacin injection for prophylaxis of endophthalmitis after phacoemulsification. Arq Bras Oftalmol 2017;80:165-7.

28 Keay L, Gower EW, Cassard SD, et al. Postcataract surgery endophthalmitis in the United States: analysis of the complete 2003 to 2004 Medicare database of cataract surgeries. Ophthalmology 2012;119:914-22.

29 O'Brien TP, Arshinoff SA, Mah FS. Perspectives on antibiotics for postoperative endophthalmitis prophylaxis: potential role of moxifloxacin. J Cataract Refract Surg 2007;33:1790-800. 